\title{
Survival between synchronous and non-synchronous multiple primary cutaneous melanomas - a SEER database analysis
}

\author{
Jie Xiong ${ }^{\text {Corresp., } 1,2}$, Yanlin Su ${ }^{3}$, Zhitong Bing ${ }^{4}$, Bihai Zhao ${ }^{\text {Corresp. } 1}$ \\ ${ }^{1}$ Department of Mathematics and Computer Science, Changsha University, Changsha, Hunan, China \\ 2 Department of Epidemiology and Health Statistics, Central South University, Changsha, Hunan, China \\ 3 Department of Gynaecology and Obstetrics, Changsha Central Hospital, Changsha, Hunan, China \\ 4 Evidence Based Medicine Center, Lanzhou University, Lanzhou, Gansu, China \\ Corresponding Authors: Jie Xiong, Bihai Zhao \\ Email address: xiongjie86@126.com, bihaizhao@163.com
}

Background. There is no criterion to distinguish synchronous and non-synchronous multiple primary cutaneous melanomas (MPMs). This study aimed to distinguish synchronous and non- synchronous MPMs and compare the survivals of them using the Surveillance, Epidemiology, and End Results database.

Methods. Synchronous and non-synchronous MPMs were distinguished by fitting the double log transformed distribution of the time interval between the first and second primary cutaneous melanomas (TIFtS) through a piecewise linear regression. The overall and melanoma-specific survivals were compared by Kaplan-Meier method and Cox proportional hazard model through modeling the occurrence of synchronous MPMs as a time dependent variable.

Results. The distribution of TIFtS was composed by three power-law distributions. And according to its first inflection point, synchronous MPMs were defined as tumors that occurred within two months. Kaplain-Meier plot revealed a significant inferior survival for synchronous MPMs than non-synchronous MPMs (P<0.0001), and the occurrence of synchronous MPM was a risk factor for overall survival of cutaneous melanoma (hazard ratio: 2.213; 95\% confidence interval: 2.087-2.346; $\mathrm{P}<0.0001$ ).

Conclusions. This study provided data analysis evidences for using two months to distinguish synchronous MPMs and non-synchronous MPMs. Furthermore, the occurrence of synchronous MPM was a risk factor for prognosis of patients with cutaneous melanoma. 
1 Survival between synchronous and non-synchronous

2 multiple primary cutaneous melanomas - a SEER

3 database analysis

4

5

6

7

8 9

Jie Xiong ${ }^{1,2}$, Yanlin $\mathrm{Su}^{3}$, Zhitong Bing ${ }^{4}$, Bihai Zhao ${ }^{1}$

${ }^{1}$ Department of Mathematics and Computer Science, Changsha University, Changsha, Hunan, China

${ }^{2}$ Department of Epidemiology and Health Statistics, Central South University, Changsha, Hunan, China

${ }^{3}$ Department of Gynaecology and Obstetrics, Changsha Central Hospital, Changsha, Hunan, China

${ }^{4}$ Evidence Based Medicine Center, Lanzhou University, Lanzhou, Gansu, China

Corresponding Author:

Bihai Zhao ${ }^{1}$ or Jie Xiong ${ }^{1,2}$

No 98 Hongshan Road, Changsha, Hunan, 410022, China

Email address: bihaizhao@163.com or xiongjie86@126.com

\section{Abstract}

Background. There is no criterion to distinguish synchronous and non-synchronous multiple primary cutaneous melanomas (MPMs). This study aimed to distinguish synchronous and nonsynchronous MPMs and compare the survivals of them using the Surveillance, Epidemiology, and End Results database.

Methods. Synchronous and non-synchronous MPMs were distinguished by fitting the double log transformed distribution of the time interval between the first and second primary cutaneous melanomas (TIFtS) through a piecewise linear regression. The overall and melanoma-specific survivals were compared by Kaplan-Meier method and Cox proportional hazard model through modeling the occurrence of synchronous MPMs as a time dependent variable.

Results. The distribution of TIFtS was composed by three power-law distributions. And according to its first inflection point, synchronous MPMs were defined as tumors that occurred within two months. Kaplain-Meier plot revealed a significant inferior survival for synchronous MPMs than non-synchronous MPMs $(\mathrm{P}<0.0001)$, and the occurrence of synchronous MPM was a risk factor for overall survival of cutaneous melanoma (hazard ratio: $2.213 ; 95 \%$ confidence interval: 2.087-2.346; $\mathrm{P}<0.0001$ ).

Conclusions. This study provided data analysis evidences for using two months to distinguish synchronous MPMs and non-synchronous MPMs. Furthermore, the occurrence of synchronous MPM was a risk factor for prognosis of patients with cutaneous melanoma. 


\section{Introduction}

41 Cutaneous melanoma (CM) is the most lethal type of skin cancer. It's incidence, mortality, and disease burden have been increasing annually (Ali et al., 2013; GBD 2015 Mortality and Causes of Death Collaborators, 2016). In 2019, it is estimated that there will be 96,480 new cases of

44 $\mathrm{CM}$ and an estimated 7,230 people will die from the disease in the United States (National Cancer Institute, 2019). Although most CMs are initially diagnosed as localized and the 5-year survival rate is high (Bradford et al., 2010), one third of all CM patients will experience disease recurrence and about $10 \%$ to $40 \%$ of patients diagnosed with localized lesions die from CM eventually (Soong et al., 1998; Hanniford et al., 2015). Therefore, it is particularly important to identify and monitor patients who have already had CM in order to detect subsequent CMs as early as possible (Ferreres et al., 2009). In the clinical research of subsequent CMs, survival comparison between patients with multiple primary CM (MPM) and single primary CM (SPM) is an old question (Hwa et al., 2012). Many studies have been carried out to address this problem but the results are controversial (Hwa et al., 2012; Utjes et al., 2017; Savoia et al., 2012). Recently, Grossman et al., revealed the potential reasons for these controversies by analyzing the Surveillance, Epidemiology, and End Results (SEER) data using a single matching method (Grossman et al., 2018).

However, MPM includes both synchronous MPM and non-synchronous MPM. Synchronous MPM is a subgroup of MPM, in which two or more primary tumors are detected simultaneously, and non-synchronous MPM is initially diagnosed as SPM until the second subsequent primary CM is detected in the follow-up. In the previous studies (Hwa et al., 2012; Utjes et al., 2017; Savoia et al., 2012; Grossman et al., 2018), synchronous MPMs were either mixed with non- synchronous MPMs or discarded. Thus, the survival of synchronous MPM is not yet known. Furthermore, more importantly, how to distinguish synchronous MPM and nonsynchronous MPM from the time interval between the first and the second primary CMs (TIFtS) is still unclear and arbitrary. Grossman et al. (2018), Pomerantz et al. (2015), and Moseley et al. (1979), adopted one year, two months, and three months to exclude synchronous MPMs, respectively. On the one hand, obviously, there is no reason to believe that two primary CMs are synchronous with each other if the second primary CM occurred one year after the first one. Thus, one year is long enough to exclude synchronous MPMs, however, a longer TIFtS may also exclude more non-synchronous MPMs. On the other hand, are two months or three months long enough to exclude synchronous MPMs? IF not long enough, this may include some synchronous MPMs.

Herein, we explored the distribution of TIFtS using the SEER database to distinguish synchronous MPM and non-synchronous MPM. Based on this distinguishment, survivals between synchronous MPM and non-synchronous MPM were compared.

\section{Materials \& Methods}

Both microscopically confirmed in situ and malignant CMs were retrieved from the SEER 18 program (1975-2016) (National Cancer Institute, 2019). The patients were followed up until December 2016. White patients with known age and at least two primary CMs were included in 
80 this study, while patients without both the first and second primary CMs were excluded.

81 Furthermore, due to the high 5-year survival rate of CM and to ensure patients have enough time 82 to develop subsequent primary CMs, patients with at least 5-years follow-up were included.

83 Thus, patients first diagnosed from 1975 to 2011 and their subsequent primary CMs occurred in 842012 to 2016 were included. Patients first diagnosed from 2012 to 2016 were excluded. Finally, 85 Patients with unknown survival time were excluded from this study. Our study was exempt from 86 institutional review board oversight, because the SEER 18 database is accessible to the public 87 and the patients in the database are de-identified.

88 We calculated the TIFtS for each patient and the distribution of TIFtS was double log 89 transformed. A piecewise linear regression, which is implemented by the "segemented" $\mathrm{R}$ package (Vito, 2008), was used to fit the double log transformed distribution. The confidential intervals of the cut points were also estimated by the "segemented" R package. Because synchronous MPMs should be near each other, thus, the first regressed line was defined as synchronous MPMs and the first cut point was defined as the optimal time to distinguish

94

95

96

97 98 synchronous and non-synchronous MPMs. Furthermore, as occurrences of subsequent primary CMs were time dependent, we modeled the occurrence of subsequent primary CM as a time dependent variable and pre-processed the survival data into a start-stop format. The validity of this approach can be derived from the counting process theory of partial likelihoods (Dirk, 2016). Finally, Overall survival and CM-specific survival were compared.

All analyses were conducted by R software (version 3.4.4) (Ihaka and Gentleman, 1996). Survivals were compared by Kaplain-Meier method and Cox proportional hazard models. $\mathrm{P}$ value $<0.05$ was considered to reject the null hypothesis.

\section{Results}

In the SEER 18 database, 128,746 CM patients diagnosed from 1975 to 2011 have developed MPMs including 187,054 primary CMs, in which 19,924 subsequent primary CMs were detected from 2012 to 2016. Furthermore, 96,910 patients didn't have both the first and the second primary CMs (112,481 tumors). After filtering, 31,836 MPM patients were firstly included in this study to investigate the distribution of TIFtS. A kernel density estimation analysis showed that the distribution of TIFtS looks like comprised by three power-law distribution (Figure 1A). Thus, we transformed the distribution of TIFtS into double log coordinates, and a piecewise linear regression was adopted to fit the double log transformed distribution. The result showed that there were three patterns that represented by three regression lines, respectively (model $\mathrm{R}$ square: 0.956, Figure 1B). For the first regression line, the inflection point was at 2 months (95\% CI: 2.53-3.72 months), and we choose this time point to distinguish synchronous and nonsynchronous MPMs. Interestingly, this agrees with the experience of Pomerantz et al (2015). and our analysis provided data analysis support for this claim.

There were two inflection points and three regression lines in the distribution of TIFtS. The second inflection point was at 93 months (95\%CI: 87.39-99.01 months), it separated the second and the third power law distributions. Although the second and third distributions were mainly patients with non-synchronous MPMs, our analysis showed a significant enrichment of patients 
120 that developed subsequent synchronous MPMs in the second power law distribution than the

121

122

123

124

125

126

127

128

129

130

131

132

133

134

135

136

137

138

139

140

141

142

143

144

145

146

147

148

149

150

151

152

153

154

155

156

157

158

third distribution $(9.1 \%$ versus $7.3 \%, \mathrm{P}<0.0001)$. Furthermore, patients in the third power law distribution were significantly younger (mean initially diagnostic age: 55.01 ) than patients in the second (mean initially diagnostic age: 61.01) and the first (mean initially diagnostic age: 60.08) distributions. Thus, the second and third distributions were termed as "older non-synchronous MPMs" and "younger non-synchronous MPMs", respectively (Figure 1B).

Actually, the indicator variable of the three power law distributions $(1,2,3$ for the first, second, and third distributions, respectively) was time dependent, because it incorporated at least the information of the second tumor, which would happen in the future. To compare the survival of synchronous and non- synchronous MPMs, we first modeled the occurrence of subsequent $\mathrm{CM}$ as a time dependent variable and pre-processed the survival data into start-stop format by the following criterion. If time intervals between a tumor and its all neighboring tumors are greater than two months, the tumor is defined as non-synchronous MPM, else, i.e., at least one neighboring tumor is within two months, the tumor is defined as synchronous MPM (Figure 2). Finally, a patient was divided into several patients according to successive occurrences of synchronous and non-synchronous MPMs (Figure 2). Because the analysis not just need the first and the second primary CMs but also need all subsequent primary CMs. We further filtered out patients that do not have complete information on subsequent primary CMs. This filtering resulted in 27,877 patients and 57,666 tumors for survival analysis. Of these patients, 10,523 were female and 17,354 were male, the average diagnostic age of the first CM was 59.88 years. At the last follow-up, 20,830 patients were alive and 7,040 were deceased, in which 2,215 deaths were caused by CM.

Univariate Cox proportional hazards model revealed that the occurrence of synchronous MPM was a risk factor for both overall survival $(\mathrm{HR}=1.808,95 \% \mathrm{CI}$ : $1.698-1.925, \mathrm{P}<0.0001)$ and CM-specific survival ( $\mathrm{HR}=1.730,95 \% \mathrm{CI}$ : $1.553-1.928, \mathrm{P}<0.0001$ ). By also modeling age of diagnosis and year of diagnosis as time dependent covariates, multivariate Cox proportional hazards model clustered by patients showed that occurrence of synchronous MPM, older age, latter diagnosis, and male were risk factors for overall survival and CM-specific survival (Table 1). Furthermore, the non-linear dose-response relationship of age at diagnosis and year of diagnosis was explored by a restricted cubic spline analysis with four knots that implemented in the R package "rms". The results showed that both age at diagnosis $(\mathrm{P}<0.001)$ and year of diagnosis $(\mathrm{P}<0.0001)$ have non-linear associations between overall survival (Figure 3 ).

However, the HR of the occurrence of synchronous MPM for overall survival was 2.371 (95\%CI: 2.108-2.371) after adjusting for age, and it was 2.213 (95\%CI: 2.087-2.3461) after adjusting for age, year, and gender. Thus, age was the main confounding factor for predicting the survival of CM patients, because it leaded to a bigger change to the HR of MPM synchrony compared to year and gender. Finally, Kaplan-Meier analysis revealed that synchronous MPMs showed a significantly inferior overall survival than non-synchronous MPMs after adjusting for age of diagnosis, year of diagnosis, and gender (Figure 4).

Peer) reviewing PDF | (2019:07:39510:1:1:NEW 13 Nov 2019) 


\section{Discussion}

160 In this study, we analyzed the distribution of TIFtS and found that the distribution could be

161

162

163

164

165

166

167

168

169

170

171

172

173

174

175

176

177

178

179

180

181

182

183

184

185

186

187

188

189

190

191

192

193

194

195

196

197

198

divided into three power-law distributions. We further define the first power-law distribution as synchronous MPMs, and its inflection point was at two months. This cut point was consistent with previous experience (Pomerantz et al. 2015), and our analysis provided data analysis support to use two months to distinguish synchronous MPMs and non- synchronous MPMs. Furthermore, survival analyses revealed that synchronous MPM was a risk factor for CM patient prognosis.

There are two ways to deal with time dependent variables to accommodate the Cox proportional hazard model. A simple way is to define a landmark time to divide patients into two groups. In this approach, patients who receive the intervention prior to the landmark go into the intervention group and those who did not are placed in the comparison group regardless of what happens in the future (Dirk, 2016). Indeed, Grossman et al.'s single matching method belongs to this kind (Grossman et al., 2018). Their landmark time is the TIFtS for each MPM patient and it varies for each patient. However, this landmark method discarded most of the patients from the analysis. The other way is to model the variable as a time dependent variable directly (Dirk, 2016). This method avoids discarding any patients and it can include all course of disease. Thus, it is better than the landmark method.

For the Cox proportional hazard model, an important assumption is the proportional hazard. In our analysis, the cumulative incidence plot for synchronous MPM was not parallel (data not shown). This revealed that the proportional hazard assumption was not satisfied. However, the cumulative incidence plot was not crossed and this indicated that although the estimated hazard ratio may be varied with time, the synchronous MPM was still a risk factor for CM patient prognosis.

In addition, pathological variables such as breslow depth, ulceration, mitosis rate, and pathological stage were not analysed due to too many missing values (Grossman et al., 2018) and inaccuracies (Mayer et al., 2017). Thus, the potential pathology of synchronous MPM needs to be illustrated in the future. Furthermore, many molecular events such as mutation (Demunter et al., 2001; Griewank et al., 2014), copy number variation (Rákosy et al., 2010; Gerami et al., 2011), epigenetic variation (Roh et al., 2016; Wouters et al., 2017), expression of genes (Brown et al., 2012; Schramm et al., 2012) and non-coding RNAs (Xiong et al., 2019; Yang et al., 2018) were reported to be involved in the prognosis of CM. Further laboratory studies aimed to investigate the potential molecular mechanisms of synchronous MPM occurrence and its prognostic roles are also in need.

\section{Conclusions}

In conclusion, this study provided data analysis evidences to distinguish synchronous and nonsynchronous MPMs. Although the occurrence of synchronous MPM was a risk factor for CM prognosis, the potential pathological and molecular mechanisms should be illustrated in the future.

\section{Acknowledgements}

Peer) reviewing PDF | (2019:07:39510:1:1:NEW 13 Nov 2019) 
199 The authors thank the SEER 18 program made the data of CM available, and all data obtained

200 from SEER keep to the rules for usage and publication of SEER. We also thank the anonymous

201 reviewers for helpful comments and suggestions. In addition, helpful discussion with Dr.

202 Yuxiang Yao from Lanzhou University is appreciated.

203 References

204 Ali Z, Yousaf N, Larkin J. 2013. Melanoma epidemiology, biology and prognosis. EJC

205 Supplements 11(2):81-91 DOI 10.1016/j.ejcsup.2013.07.012.

206 Bradford PT, Freedman DM, Goldstein AM, Tucker MA. 2010. Increased Risk of Second

207 Primary Cancers After a Diagnosis of Melanoma. Arch Dermatol 146(3):265-272 DOI

208 10.1001/archdermatol.2010.2.

209 Brown ER, Doig T, Anderson N, Brenn T, Doherty V, Xu Y, Bartlett JM, Smyth JF,

210 Melton DW. 2012. Association of galectin-3 expression with melanoma progression and

211 prognosis. Eur J Cancer 48(6):865-874 DOI 10.1016/j.ejca.2011.09.003.

212 Demunter A, Ahmadian MR, Libbrecht L, Stas M, Baens M, Scheffzek K, Degreef H, De

213 Wolf-Peeters C, van Dan Oord JJ. 2001. A Novel N-ras Mutation in Malignant Melanoma Is

214 Associated with Excellent Prognosis. Cancer Res 61(12):4916-22 DOI 10.1002/1097-

215 0142(20010615)91:12<2476::AID-CNCR1283>3.0.CO;2-Z.

216 Dirk FM. 2016. Applied Survival Analysis Using R. 1st ed. Switzerland, Springer.

217 Ferreres JR, Moreno A, Marcoval J. 2009. Multiple Primary Melanoma. Actas Dermosifiliogr

218 100(5):414-419.

219 GBD 2015 Mortality and Causes of Death Collaborators. 2016. Global, regional, and national

220 life expectancy, all-cause mortality, and cause-specific mortality for 249 causes of death, 1980-

221 2015: a systematic analysis for the Global Burden of Disease Study 2015. Lancet

222 388(10053):1459-1544 DOI 10.1016/S0140-6736(16)31012-1.

223 Gerami P, Jewell SS, Pouryazdanparast P, Wayne JD, Haghighat Z, Busam KJ,

224 Rademaker A, Morrison L. 2011. Copy Number Gains in 11q13 and 8q34 Are Highly Linked

225 to Prognosis in Cutaneous Malignant Melanoma. J Mol Diagn 13(3):352-358 DOI

226 10.1016/j.jmoldx.2011.01.011.

227 Griewank KG, Murali R, Puig-Butille JA, Schilling B, Livingstone E, Potrony M, Carrera

228 C, Schimming T, Möller I, Schwamborn M, Sucker A, Hillen U, Badenas C, Malvehy J,

229 Zimmer L, Scherag A, Puig S, Schadendorf D. 2014. TERT promoter mutation status as an

230 independent prognostic factor in cutaneous melanoma. $J$ Natl Cancer Inst 106(9) DOI

231 10.1093/jnci/dju246.

232 Grossman D, Farnham JM, Hyngstrom J, Klapperich ME, Secrest AM, Empey S. 2018.

233 Similar survival of patients with multiple vs. single primary melanomas based on Utah SEER

234 data (1973-2011). J Am Acad Dermatol 79(2):238-244 DOI 10.1016/j.jaad.2018.02.055.

235 Hanniford D, Zhong J, Koetz L, Gaziel-Sovran A, Lackaye DJ, Shang S, Pavlick A,

236 Shapiro R, Berman R, Darvishian F, Shao Y, Osman I, Hernando E. 2015. A miRNA-Based

237 Signature Detected in Primary Melanoma Tissue Predicts Development of Brain Metastasis. Clin

238 Cancer Res 21(21):4903-4912 DOI 10.1158/1078-0432.CCR-14-2566. 
239 Hwa C, Price LS, Belitskaya-Levy I, Ma MW, Shapiro RL, Berman RS, Kamino H,

240 Darvishian F, Osman I, Stein JA. 2012. Single versus multiple primary melanomas: old 241 questions and new answers. Cancer 118(17):4184-4192 DOI 10.1002/cncr.27407.

242 Ihaka R, Gentleman R. 1996. R: A language for data analysis and graphics. J Comput Gr Stat 243 5(3):299-314 DOI 10.2307/1390807.

244 Mayer JE, Fathi R, Norris DA. 2017. Inaccuracies in SEER registry data on melanoma 245 thickness. J Am Acad Dermatol 77(1):e17 DOI 10.1016/j.jaad.2017.01.061.

246 Moseley HS, Giuliano AE, Storm FK, Clark WH, Robinson DS, Morton DL. 1979. Multiple 247 primary melanoma. Cancer 43(3):939-944 DOI 10.1002/1097-0142(197903)43:3<939::aid-

248 cncr2820430323>3.0.co;2-n.

249 National Cancer Institute. Surveillance, Epidemiology, and End Results program: about the 250 SEER program. Available from: http://seer.cancer.gov/. Accessed March 6, 2019.

251 Pomerantz H, Huang D, Weinstock MA. 2015. Risk of subsequent melanoma after melanoma 252 in situ and invasive melanoma: A population-based study from 1973 to 2011. J Am Acad 253 Dermatol 72(5):794-800 DOI 10.1016/j.jaad.2015.02.006.

254 Rákosy Z, Vízkeleti L, Ecsedi S, Vokó Z, Bégány A, Barok M, Krekk Z, Gallai M, 255 Szentirmay Z, Adány R, Balázs M. 2010. EGFR gene copy number alterations in primary 256 cutaneous malignant melanomas are associated with poor prognosis. Int J Cancer 121(8): 1729-

2571737 DOI 10.1002/ijc.22928.

258 Roh MR, Gupta S, Park KH, Chung KY, Lauss M, Flaherty KT, Jönsson G, Rha SY, Tsao 259 H. 2016. Promoter Methylation of PTEN is a Significant Prognostic Factor in Melanoma 260 Survival. J Invest Dermatol 136(5):1002-1011 DOI 10.1016/j.jid.2016.01.024.

261 Savoia P, Osella-Abate S, Deboli T, Marenco F, Stroppiana E, Novelli M, Fierro MT, 262 Bernengo MG. 2012. Clinical and prognostic reports from 270 patients with multiple primary 263 melanomas: a 34-year single-institution study. J Eur Acad Dermatol Venereol 26(7):882-888 264 DOI 10.1111/j.1468-3083.2011.04181.x.

265 Schramm SJ, Campain AE, Scolyer RA, Yang YH, Mann GJ. 2012. Review and Cross266 Validation of Gene Expression Signatures and Melanoma Prognosis. J Invest Dermatol 267 132(2):274-283 DOI 10.1038/jid.2011.305.

268 Soong SJ, Harrison RA, Mccarthy WH, Urist MM, Balch CM. 1998. Factors affecting 269 survival following local, regional, or distant recurrence from localized melanoma. J Surg Oncol 270 67(4):228-233 DOI 10.1002/(sici)1096-9098(199804)67:4<228::aid-jso4>3.0.co;2-a.

271 Utjes D, Lyth J, Lapins J, Eriksson H. 2017. Reduced disease-specific survival following a 272 diagnosis of multiple primary cutaneous malignant melanomas-a nationwide, population-based 273 study. Int J Cancer 141(11):2243-2252 DOI 10.1002/ijc.30925.

274 Vito MR Muggeo. 2008. segmented: an R Package to Fit Regression Models with Broken-Line 275 Relationships. R News 8(1):20-25.

276 Wouters J, Vizoso M, Martinez-Cardus A, Carmona FJ, Govaere O, Laguna T, Joseph J, 277 Dynoodt P, Aura C, Foth M, Cloots R, van den Hurk K, Balint B, Murphy IG, McDermott 278 EW, Sheahan K, Jirström K, Nodin B, Mallya-Udupi G, van den Oord JJ, Gallagher WM, 
279 Esteller M. 2017. Comprehensive DNA methylation study identifies novel progression-related 280 and prognostic markers for cutaneous melanoma. BMC Med 15(1):101 DOI 10.1186/s12916281 017-0851-3.

282 Xiong J, Bing Z, Guo S. 2019. Observed Survival Interval: A Supplement to TCGA Pan-Cancer 283 Clinical Data Resource. Cancers 11(3):e280 DOI 10.3390/cancers11030280.

284 Yang S, Xu J, Zeng X. 2018. A six-long non-coding RNA signature predicts prognosis in 285 melanoma patients. Int J Oncol 52(4):1178-1188 DOI 10.3892/ijo.2018.4268. 


\section{Table $\mathbf{1}$ (on next page)}

Multivariate Cox proportional hazard model clustered by patients.

$\mathrm{HR}$, hazard ratio; $\mathrm{Cl}$, confidence interval; OS, overall survival; CMSS, cutaneous melanoma specific survival; MPM, multiple primary cutaneous melanoma. 
1 Table 1. Multivariate Cox proportional hazard model clustered by patients.

\begin{tabular}{lllllll}
\hline & $\mathrm{HR}^{\mathrm{OS}}$ & $95 \% \mathrm{CI}^{\mathrm{OS}}$ & $\mathrm{POS}$ & HR $^{\mathrm{CMSS}}$ & $95 \% \mathrm{CI}^{\mathrm{CMSS}}$ & $\mathrm{P}^{\mathrm{CMSS}}$ \\
\hline Synchronous MPM & 2.213 & $2.087-2.346$ & $<0.0001$ & 1.980 & $1.776-2.207$ & $<0.0001$ \\
Age at diagnosis & 1.088 & $1.086-1.091$ & $<0.0001$ & 1.054 & $1.050-1.059$ & $<0.0001$ \\
Year of diagnosis & 0.993 & $0.990-0.996$ & $<0.0001$ & 0.987 & $0.979-0.995$ & 0.001 \\
Sex & 1.341 & $1.277-1.408$ & $<0.0001$ & 1.427 & $1.301-1.566$ & $<0.0001$ \\
\hline
\end{tabular}

2 HR, hazard ratio; CI, confidence interval; OS, overall survival; CMSS, cutaneous melanoma

3 specific survival; MPM, multiple primary cutaneous melanoma. 


\section{Figure 1}

Distribution of TIFtS.

Kernal density estimation of the distribution of TIFtS (A). Piece wise linear regression analysis for the double log transformed distribution of TIFtS (B). The solid line, dashed line, and dotted line are three regression lines that represent synchronous MPMs, older non- synchronous MPMs, and younger non- synchronous MPMs, respectively. The numbers in the brackets are intercepts and slopes of the regression lines. MPM, multiple primary cutaneous melanoma; TIFtS, time interval between the first and the second primary cutaneous melanomas.
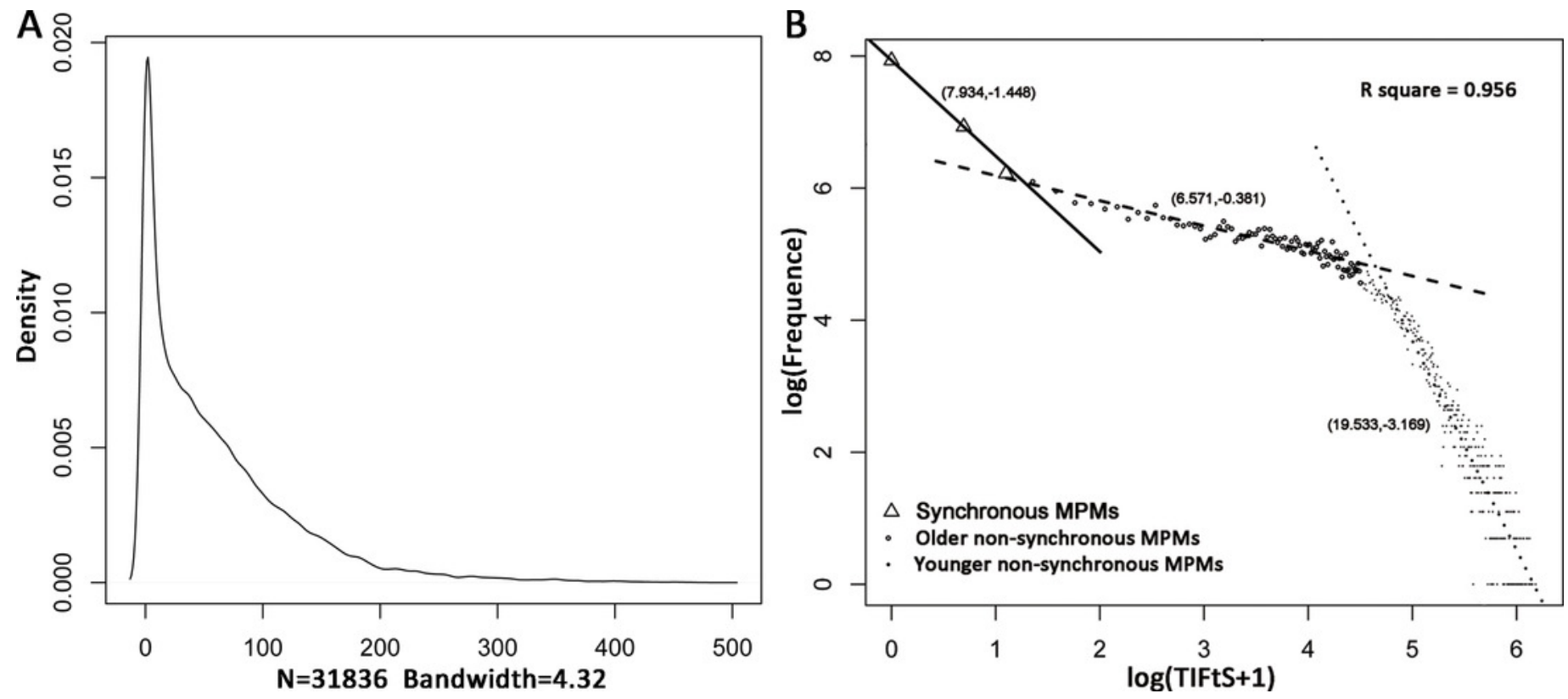


\section{Figure 2}

Pre-processing of the survival data.

Star, number in the star, solid dot, and hollow dot represent tumor, tumor sequence number, death, and censored, respectively. Tumor 1 and 2 are non-synchronous MPMs, and tumor 3 and 4 are synchronous MPMs. Patient $A$ is divided into three patients, the first one starts from the occurrence of tumor1 and ends up at the occurrence of tumor2; the second one starts from the occurrence of tumor 2 and ends up at the occurrence of tumor 3 ; the last one starts from the occurrence of tumor 3 and ends up until death. MPM, multiple primary cutaneous melanoma. 


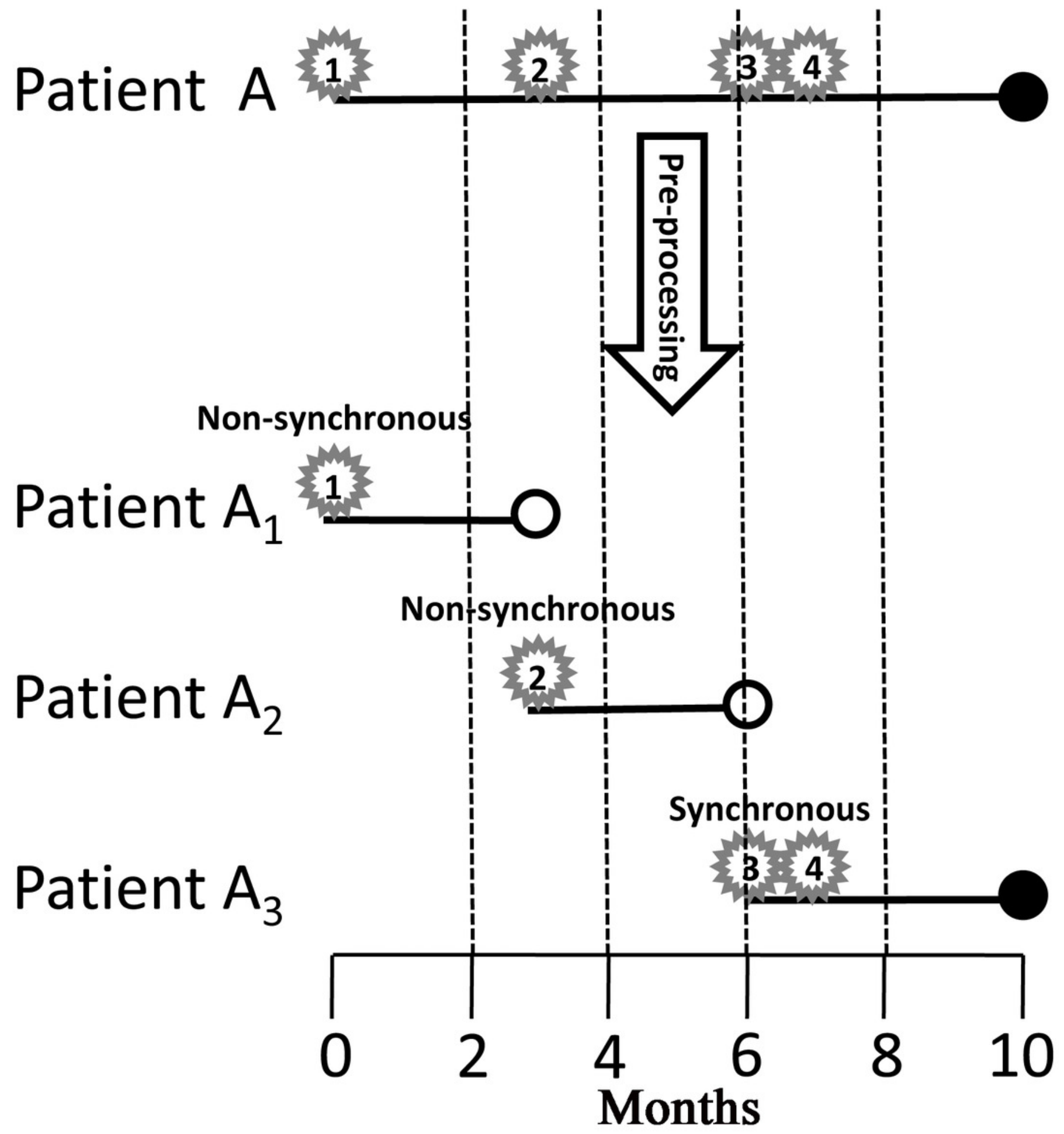


Figure 3

Non-linear dose-response relationships.

Restricted cubic spline analysis of the association between overall survival and age of diagnosis (A), and the association between overall survival and year of diagnosis (B). The middle solid line indicates the point estimates of hazard ratios and the broken lines indicate the lower and upper limits of the corresponding 95\% confidence intervals. Four knots were used for the analysis.

A

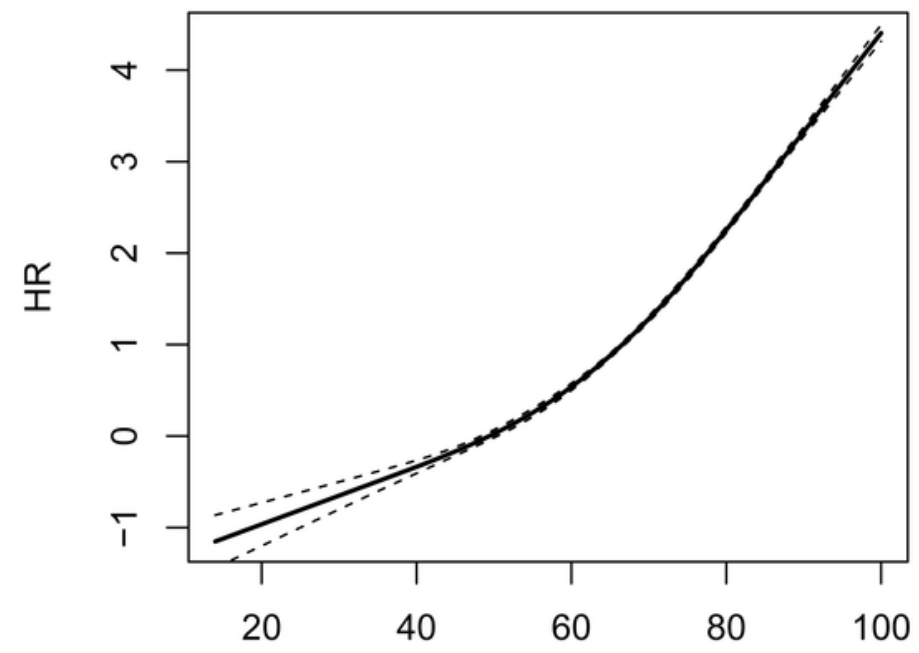

Age at diagnosis
B

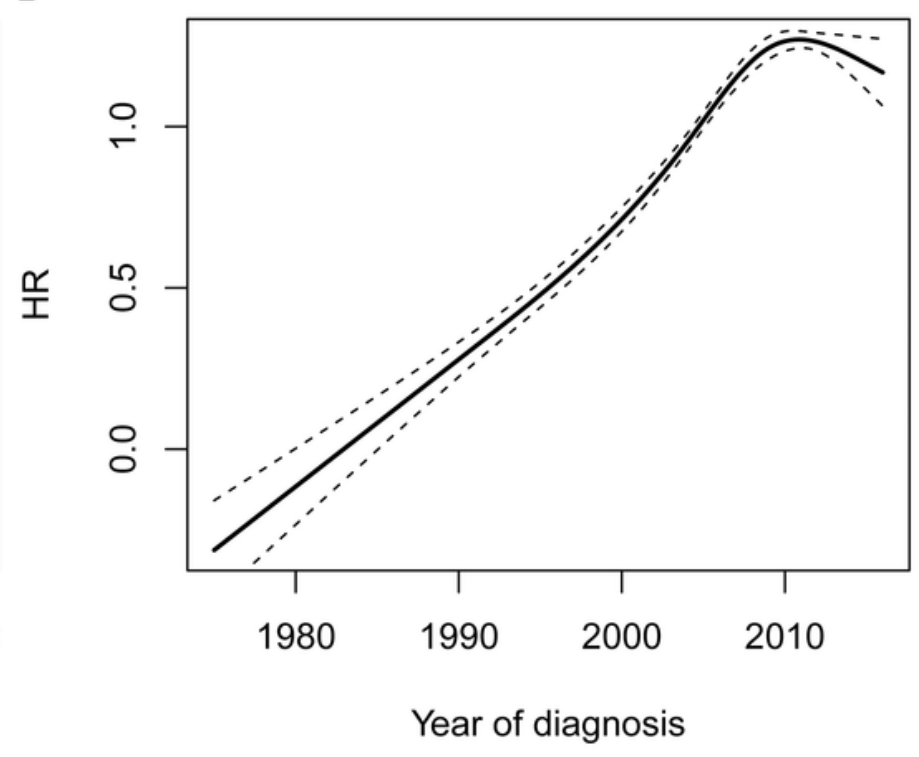


Figure 4

Kaplain-Merier curves of synchronous and non-synchronous MPMs.

Synchronous MPMs showed a significantly inferior overall survival than non-synchronous MPMs after adjusting for age of diagnosis, year of diagnosis, and gender. MPM, multiple primary cutaneous melanoma.

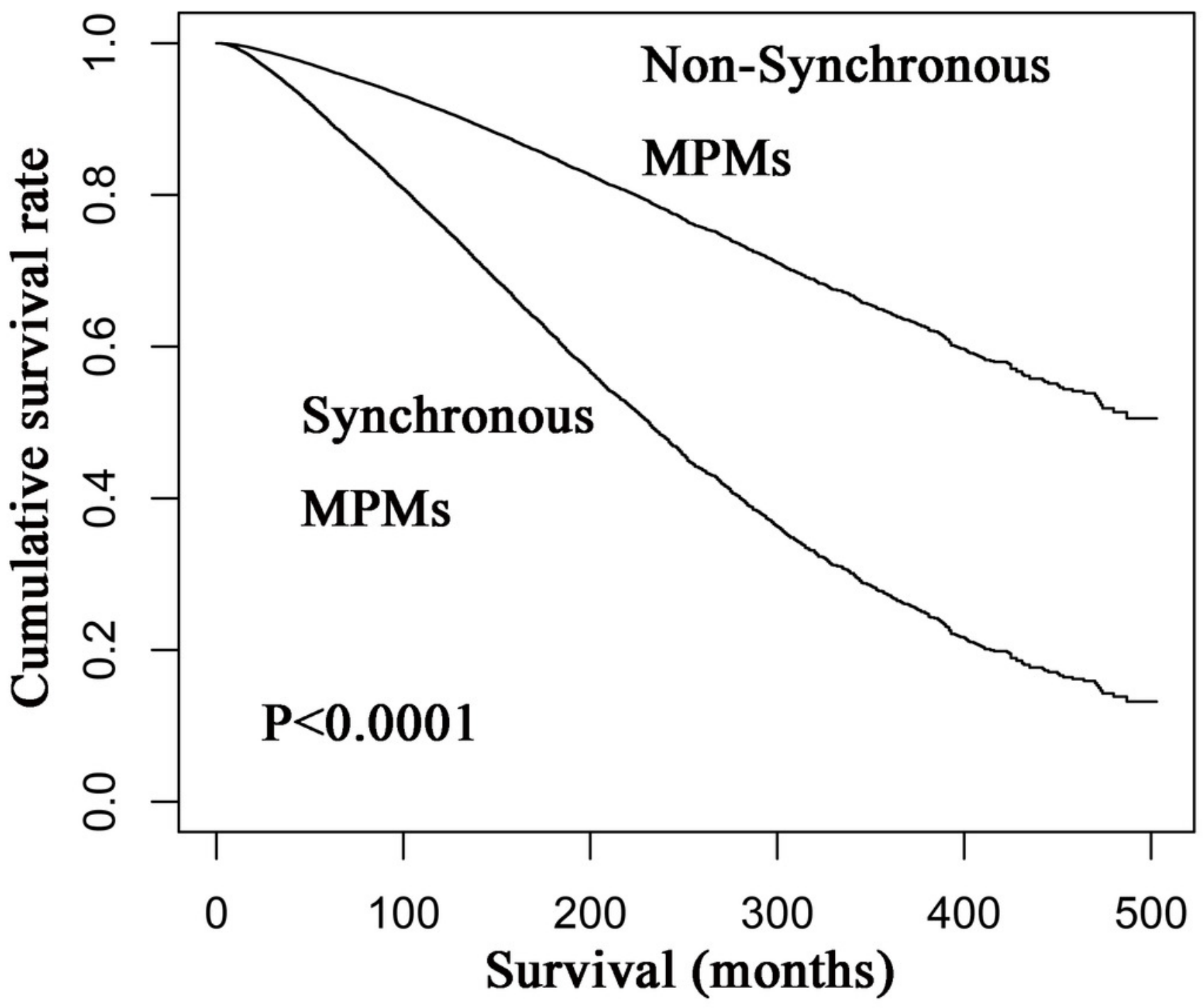

